\title{
Strains of Escherichia coli 0157:H8 from human diarrhoea belong to attaching and effacing class of $E$ coli
}

\author{
S M Scotland, G A Willshaw, T Cheasty, B Rowe
}

\begin{abstract}
Aims: To determine whether 17 Escherichia coli 0157:H8 strains isolated from patients with diarrhoea in the United Kingdom were putative pathogens.

Methods: The strains had been isolated by the use of 0157 antiserum, available for the detection of Vero cytotoxin (VT) producing strains of $E$ coli 0157 that are usually of flagellar (H) type 7, but may also be nonmotile. The strains were examined for VT production, for their ability to adhere to HEp-2 cells, and for hybridisation with several DNA probes that recognise pathogenic properties of $E$ coli. Their ability to ferment sorbitol and to produce $\beta$-glucuronidase was also investigated, as these tests are used to discriminate VT positive 0157 strains.

Results: The 0157:H8 strains did not produce VT. All gave localised attachment to HEp-2 cells, associated with a positive fluorescence-actin staining test, and all hybridised with the $E$ coli attaching and effacing (eae) probe. In addition to the difference in VT production, 0157:H8 strains could be distinguised from VT positive 0157 strains by their $\beta$-glucuronidase activity, their failure to produce enterohaemolysin, and their lack of hybridisation with the CVD419 probe derived from a plasmid in an 0157:H7 strain.
\end{abstract}

Conclusions: The 0157:H8 strains had in vitro properties characteristic of the class of $E$ coli that causes attaching and effacing lesions in epithelial intestinal cells. They may therefore be considered a putative cause of diarrhoea but their prevalence remains to be established. Several 0157:H8 strains failed to ferment sorbitol in agar plates and therefore could be misidentified as VT positive 0157 strains. Confirmatory tests for VT production are needed when 0157 strains are isolated from faeces.

(F Clin Pathol 1992;45:1075-1078)

Division of Enteric Pathogens, Central Public Health

Laboratory, 61

Colindale Avenue

London NW9 5HT

$S M$ Scotland

G A Willshaw

T Cheasty

B Rowe

Correspondence to:

Sylvia Scotland

Accepted for publication

18 May 1992

Escherichia coli serogroup 0157 encompasse strains belonging to many different flagellar $\mathrm{H}$ types and with differing pathogenic properties. Some $E$ coli 0157 strains cause porcine colibacillosis or extra-intestinal infections in man. ${ }^{12}$ In recent years the most important development has been the recognition that strains of serotype 0157 are an important cause of haemorrhagic colitis and haemolytic uraemic syndrome in North America and the United Kingdom. ${ }^{3}$ These strains produce one or more Vero cytotoxins (VT) and carry a plasmid of molecular size about 60 megadaltons; such strains have been termed enterohaemorrhagic $E$ coli (EHEC). ${ }^{4}$ The VT positive strains of $E$ coli 0157 are usually of $H$ type 7 , but nonmotile strains have also been reported. Unlike most strains of $E$ coli, most VT positive strains of serogroup 0157 do not ferment sorbitol within one day. ${ }^{5}$ Sorbitol MacConkey agar (SMAC agar) which contains sorbitol in place of lactose makes use of this characteristic and may be used for faecal culture and the recognition of these organisms. ${ }^{6}$ Pale colonies after overnight incubation must be confirmed biochemically as $E$ coli, and antiserum, usually in the form of latex agglutination kits, is used to confirm that they are serogroup $0157 .^{7}$ Notwithstanding this, there have been reports of atypical sorbitol fermenting VT positive 0157 strains from Germany and the United States. $^{89}$

The Division of Enteric Pathogens, in its role as a national reference facility, has received $E$ coli 0157 strains isolated from patients for the confirmation of VT production and determination of VT type and phage type for epidemiological purposes. Most of these strains were indeed VT positive 0157 strains, but a small number of VT negative 0157 strains were received and most of these were $\mathrm{H}$ type 8. Because the 0157:H8 strains were isolated from patients with intestinal disease we examined them for several relevant pathogenic properties. These included tests for attachment to cells grown in culture. In some enteropathogenic $E$ coli (EPEC) the ability to cause clinically important diarrhoea in volunteer studies and good attachment in vitro is dependent on plasmid encoded genes. The 0157:H8 strains were tested for hybridisation with the EPEC adherence factor (EAF) probe developed from one such plasmid. ${ }^{10}$ The fluorescence-actin staining (FAS) test ${ }^{11}$ which correlates with the ability to cause attaching and effacing (AE) lesions of the intestinal microvilli, was performed and also hybridisation with the eae probe derived from chromosomal sequences necessary for the ability to cause $\mathrm{AE}$ lesions. $^{12}$

\section{Methods}

$E$ coli 0157:H8 strains were isolated from faecal specimens of 17 patients with diarrhoea 
in the United Kingdom; all had been isolated since 1984, with 11 strains isolated in 1990 or 1991. Twelve patients were aged between 1 and 15 months, one was aged 3 years, one was aged 16 years and the age of three patients was not given. Although other clinical details were rarely provided, the diarrhoea was stated to be bloody in one instance and accompanied by vomiting in two. Diarrhoea in the child aged 3 years had persisted for one month when the faecal specimen was taken. The patient aged 16 years had abnormal renal function; the diarrhoea was stated as being non-bloody.

Serotyping was performed with antisera against $173 \mathrm{O}$ antigens and $56 \mathrm{H}$ antigens using standard procedures. ${ }^{13}$ Strains were tested for the ability to ferment sorbitol or rhamnose in Andrade peptone waters (containing $0.5 \%$ of the substrate). Colonial appearance was examined on SMAC agar and on SMAC agar containing rhamnose $(0.5 \%)$ and cefixime $(0.05 \mathrm{mg} / \mathrm{l})$ (CR-SMAC), as described by Chapman et al. ${ }^{14}$ Production of glucuronidase was assessed by the ability to hydrolyse 4-methylumbelliferyl- $\beta$-Dglucuronide (MUG) using a previously described method. ${ }^{15}$ Production of enterohaemolysin was tested on agar containing washed sheep erythrocytes. ${ }^{16}$ All the strains were phage-typed using the extended scheme forVT positive $E$ coli 0157 strains. $^{17}$

Strains were tested for the ability to produce Vero cytotoxin using the Vero cell test and for adhesion to HEp-2 cells, using a six hour test in which the cells were washed after the first three hour period. ${ }^{18}$ For the FAS test cells were fixed with formalin, permeabilised with Triton $\mathrm{X}-100$, and treated with fluorescein isothiocyanate phalloidin. ${ }^{11}$ The pattern of attachment was assessed using phase contrast microscopy ${ }^{1920}$ and attached bacteria were related to the appearance of fluorescing condensed actin using ultraviolet light. ${ }^{11}$

Strains were tested by colony hybridisation ${ }^{21}$ for the presence of genes that may be associated with virulence. The probe for VT1 sequences was a 0.75 kilobase $H$ incII fragment derived from a phage in strain $\mathrm{H} 19$ (serotype $026: \mathrm{H} 11)^{22}$ and that for VT2 was a 0.85 kilobase SmaI-PstI fragment derived from the VT2 encoding phage of strain E32511 (serotype 0157:H non-motile). ${ }^{23}$ The possession of sequences associated with $\mathrm{AE}$ lesions was detected with a 1 kilobase $K p n I-S a l I$ fragment, the eae probe. ${ }^{12}$ To test for the presence of EPEC adherence factor (EAF) the EAF probe, a 1 kilobase Sall-BamHI fragment, was used. ${ }^{10}$ All strains were also tested with the CVD419 probe, a 3.4 kilobase HindIII fragment of the 60 megadalton plasmid present in $E$ coli 0157:H7 strains. ${ }^{4}$ Hybridisation and washing for radioactive probes were as described previously. ${ }^{22}$ The CVD419 probe was labelled with digoxigenin and used under the conditions described by Thomas et al. ${ }^{24}$

Strains were examined for plasmid DNA by gel electrophoresis of plasmid DNA prepared by the method of Birnboim and Doly. ${ }^{25}$ Molecular sizes were measured relative to standard plasmids run on the same gel.

\section{Results}

The results of tests with the 0157:H8 strains are shown in the table. Results deduced from published findings for similar tests with 0157:H7 strains are given for comparison.

All the 0157:H8 strains fermented sorbitol and rhamnose by the tube method within 24 hours. However, on SMAC medium, after 24 hours the appearance of the colonies varied: two strains gave colourless colonies, two gave pale pink colonies, while the remaining 13 strains gave dark red colonies. On CR-SMAC all colonies were dark red. The strains did not produce enterohaemolysin. All showed $\beta$-glucuronidase activity.

The $17 E$ coli 0157:H8 strains did not produce Vero cytotoxin in a tissue culture test or hybridise with probes specific for VT1 or VT2. All strains produced localised attachment to HEp-2 cells. The clusters contained more than 50 bacteria and the percentage of cells with such clusters ranged from 1-96, with a mean of 20 . In the FAS test clusters were associated with the intense fluorescence indicative of a positive test. The strains hybridised with the eae probe but not with the EAF probe.

All 17 strains gave the same pattern of lytic reactions with the phages used in the typing scheme for VT positive $E$ coli 0157 strains. The pattern did not match that of any of the 62 published phage-types of 0157 strains.

All the 0157:H8 strains carried a plasmid in the size range 72-78 megadaltons; additional plasmids from 38-50 megadaltons were present in four strains and a further three strains had small plasmids less than 2 megadaltons in size. The strains did not hybridise with the CVD419 probe.

\section{Discussion}

The 0157:H8 strains had been referred to the DEP for VT testing because they had agglutinated with 0157 antiserum even though 13 of them should have produced red colonies on SMAC agar. Confluent or semi-confluent growth of fermenting strains on SMAC agar may often appear pale pink or colourless and

Properties of 17 strains of $E$ coli 0157:H8 isolated from humans with diarrhoea and a comparison with properties of 0157:H7 strains as deduced from published reports*

\begin{tabular}{lll}
\hline & \multicolumn{2}{c}{ Properties of strains of serotype } \\
\cline { 2 - 3 } & $0157: H 8$ & $0157: H 7$ \\
\hline $\begin{array}{l}\text { Fermentation of sorbitol (1 day) } \\
\text { Peptone water }\end{array}$ & + & - \\
SMAC agar & $+(15) ;-(2)$ & - \\
CR-SMAC agar & + & - \\
Production of & - & + \\
VT & - & + \\
Enterohaemolysin & + & - \\
$\beta$-glucuronidase & LA & LAt \\
Adhesion to HEp-2 cells & + & + \\
Reaction in FAS test & & + \\
Hybridisation with probes for & - & + \\
VT1 and/or VT2 & - & + \\
EAF & + & + \\
eae & + & +
\end{tabular}

*Data from references $46111214-10233$,

tData from references" and ${ }^{26}$; other adhesion results were reported in reference 27 . 
this may cause confusion. Two strains did produce colourless colonies on SMAC agar and so may well have been mistaken for VT positive 0157 strains. Only the VT positive 0157 strains would have appeared colourless after 24 hours on the recently described CRSMAC medium.

The 0157:H8 strains produced localised adhesion to HEp-2 cells, were positive in the FAS test, and hybridised with the eae probe. Strains with these properties are considered to belong to the attaching and effacing class of $E$ coli (AEEC) ${ }^{28}$ which includes both EPEC and EHEC such as VT producing strains of 0157:H7. ${ }^{11229}$ These attaching and effacing but VT negative $E$ coli $0157:$ H8 strains may represent another group of 0157 strains that are a putative cause of diarrhoea. Further studies are needed to assess the prevalence of such strains.

We have reported other strains of $E$ coli that have the properties of AEEC but, like the 0157:H8 strains, do not hybridise with the EAF probe. These include VT negative strains belonging to traditional EPEC serogroups such as $0128,{ }^{3031}$ and VT positive strains belonging to serogroups 05, 026, and $0103 .^{30}{ }^{32}$ For several of these EAF negative strains, for example, E25253 (0128:H2) and S22-1 (0103:H2), loss of a plasmid resulted in a decrease in attachment to HEp-2 cells, although adhering bacteria still caused actin accumulation. ${ }^{3031}$ These results indicate that, as for EAF positive strains such as E2348/69 (0127:H6), ${ }^{10}$ plasmid-encoded genes may have a role in the attachment of EAF negative strains. Similar results have been reported in some studies with EAF negative, VT positive 0157:H7 strains which carry the common 60 megadalton plasmid. ${ }^{27}$ We do not know yet whether the 70 megadalton plasmid common to the EAF negative, VT negative 0157:H8 strains is similarly involved in attachment.

The 0157:H8 strains differed from VT positive 0157 strains in several respects. Enterohemolysin was produced by all $32 \mathrm{VT}$ positive 0157 strains tested in the study of Beutin et $a l,{ }^{16}$ but the eight $0157:$ H8 strains did not produce enterohemolysin. Unlike most $E$ coli strains, VT positive 0157 strains do not produce $\beta$-glucuronidase. The 0157:H8 strains resembled other VT negative 0157 strains $^{1533}$ in possessing $\beta$-glucuronidase activity. However, the VT positive non-motile 0157 strains examined by Karch et al, ${ }^{9}$ that were atypically sorbitol fermenting, were also $\beta$-glucuronidase positive. The 0157:H8 strains did not hybridise with the CVD419 probe. From this result it could be concluded that the large 70 megadalton plasmid carried by these strains did not have a gene sequence homologous to CVD419 that was originally derived from the large 60 megadalton plasmid carried by an 0157:H7 strain.

The availability of 0157 antiserum for the investigation of cases of diarrhoea for the presence of VT positive strains of 0157 has occasionally resulted in the isolation of VT negative 0157:H8 strains. The presence of these strains in the faeces of patients with diarrhoea reinforces the advice that 0157 strains should be sent to a reference laboratory for confirmatory tests such as VT production.

1 Scotland SM, Willshaw GA, Smith HR, Rowe B. Properties of strains of Escherichia coli belonging to serogroup 0157 with special reference to production of Vero cytotoxins VT1 and VT2. Epidemiol Infect 1987;99:613-24.

2 Whittam TS, Wilson RA. Genetic relationships among pathogenic Escherichia coli of serogroup 0157. Infect Immun 1988;56:2467-73.

3 Karmali MA. Infection by Vero cytotoxin-producing Escherichia coli. Clin Microbiol Rev 1989;2:15-38.

4 Levine MM, Xu J-g, Kaper JB, et al. A DNA probe to identify enterohemorrhagic Escherichia coli of 0157:H7 and other serotypes that cause hemorrhagic colitis and hemolytic uremic syndrome. $f$ Infect Dis 1987; 156:175-82.

5 Wells JG, Davis BR, Wachsmuth IK, et al. Laboratory investigation of hemorrhagic colitis outbreaks associated with a rare Escherichia coli serotype. F Clin Microbiol 1983;18:512-20.

6 March SB, Ratnam S. Sorbitol-MacConkey medium for detection of Escherichia coli 0157:H7 associated with detection of Escherichia coli 0157:H7 associated with

7 March SB, Ratnam S. Latex agglutination test for detection of Escherichia coli serotype 0157. I Clin Microbiol 1989;27:1675-7.

8 Farmer JJ, Davis BR. H7 antiserum-sorbitol fermentation medium: a single tube screening medium for detecting Escherichia coli 0157:H7 associated with hemorrhagic colitis. I Clin Microbiol 1985;22:620-5.

9 Karch H, Wiss R, Gloning H, Emmrich P, Aleksic S, Bockemuhl J. Hamolytisch-uramisches syndrom bei kleinkindern durch Verotoxin-produzierende Escherichia coli. Disch Med Wschr 1990;115:489-95.

10 Baldini MM, Nataro JP, Kaper JB. Localization of a determinant for HEp-2 adherence by enteropathogenic determinant for HEp-2 adherence by enterop

11 Knutton S, Baldwin T, Williams PH, McNeish AS. Actin accumulation at sites of bacterial adhesion to tissue culture cells: basis of a new diagnostic test for enteropathogenic and enterohemorrhagic Escherichia coli. Infect Immun 1989;57:1290-8.

12 Jerse AE, Yu J, Tall BD, Kaper JB. A genetic locus of enteropathogenic Escherichia coli necessary for the production of attaching and effacing lesions on tissue culture cells. Proc Natl Acad Sci USA 1990;87:7839-43.

13 Gross RJ, Rowe B. Serotyping of Escherichia coli. In: Sussman M, ed. The virulence of Escherichia coli: reviews and methods. London:Academic Press, 1985:345-63.

14 Chapman PA, Siddons CA, Zadik PM, Jewes L. An improved selective medium for the isolation of Escherichia coli 0157. F Med Microbiol 1991;35:107-10.

15 Scotland SM, Cheasty T, Thomas A, Rowe B. Betaglucuronidase activity of Vero cytotoxin-producing strains of Escherichia coli, including serogroup 0157, isolated in the United Kingdom. Lett Appl Microbiol 1991;13:42-4.

16 Beutin L, Montenegro MA, Orskov I, et al. Close association of verotoxin (Shiga-like toxin) production with enterohemolysin production in strains of Escherichia coli. F Clin Microbiol 1989;27:2559-64.

17 Khakria R, Duck D, Lior H. Extended phage-typing scheme for Escherichia coli 0157:H7. Epidemiol Infect 1990;105:511-20.

18 Scotland SM, Gross RJ, Rowe B. Laboratory tests for enterotoxin production, enteroinvasion and adhesion in diarrhoeagenic Escherichia coli. In: Sussman M, ed. The virulence of Escherichia coli: reviews and methods. London: virulence of Escherichia coli: reviews

19 Cravioto A, Gross RJ, Scotland SM, Rowe B. An adhesive factor found in strains of Escherichia coli belonging to the traditional infantile enteropathogenic serotypes. Curr Microbiol 1979;3:95-9.

20 Scaletsky ICA, Silva MLM, Trabulsi LR. Distinctive patterns of adherence of enteropathogenic Escherichia coli to HeLa cells. Infect Immun 1984;45:534-6.

21 Maniatis T, Fritsch EF, Sambrook J. Molecular cloning: a laboratory manual. Cold Spring Harbor, New York: Cold Spring Harbor Laboratory, 1982.

22 Willshaw GA, Smith HR, Scotland SM, Rowe B. Cloning of genes determining the production of Vero cytotoxin by Escherichia coli. $\mathcal{F}$ Gen Microbiol 1985;131:3047-53.

23 Willshaw GA, Smith HR, Scotland SM, Field AM, Rowe B. Heterogeneity of Escherichia coli phages encoding Vero cytotoxins: comparison of cloned sequences determining VT1 and VT2 and development of specific gene probes. $\mathcal{F}$ Gen Microbiol 1987;133:1309-17.

24 Thomas A, Smith HR, Willshaw GA, Rowe B. Nonradioactively labelled polynucleotide and oligonucleotide DNA probes, for selectively detecting Escherichia coli strains producing Vero cytotoxins VT1, VT2 and VT2 variant. Mol Cell Probes 1991;5:129-35.

25 Birnboim HC, Doly J. A rapid alkaline extraction procedure for screening recombinant plasmid DNA. Nucleic Acids Res 1979;7:1513-23.

26 Sherman P, Soni R, Petric M, Karmali M. Surface properties of the Vero cytotoxin-producing Escherichia coli 0157:H7. Infect Immun 1987;55:1824-9. 
27 Toth I, Cohen ML, Rumschlag HS, et al. Influence of the 60 megadalton plasmid on adherence of Escherichia coli 0157: 7 and genetic derivatives. Infect Immun 1990;58:1223-31

28 Moon HW, Whipp SC, Argenzio RA, Levine MM, Giannella RA. Attaching and effacing activities of rabbit and human enteropathogenic Escherichia coli in pig and human enteropathogenic Escherichia coli in pig

29 Tzipori S, Karch H, Wachsmuth KI, zipori S, Karch H, Wachsmuth KI, et al. Role of a 60 megadalton plasmid and Shiga-like toxins in the pathogenesis of infection caused by enterohemorrhagic Escherichia coli $0157: \mathrm{H} 7$
$1987 ; 55: 3117-25$

30 Scotland SM, Willshaw GA, Smith HR, Rowe B. Properties of strains of Escherichia coli 026:H11 in relation to their enteropathogenic or enterohemorrhagic classification. $\mathcal{F}$
Infect Dis 1990;162:1069-74.

31 Scotland SM, Smith HR, Rowe B. Escherichia coli 0128 strains from infants with diarrhea commonly show localized adhesion and positivity in the fluorescent-actin staining test but do not hybridize with an enteropathostaining test but do not hybridize with an enteropathogenic E coli adher

32 Hall GA, Dorn CR, Chanter N, Scotland SM, Smith HR, Rowe B. Attaching and effacing lesions in vivo and adhesion to tissue culture cells of Vero-cytotoxin-producing Escherichia coli belonging to serogroups 05 and 0103. f Gen Microbiol 1990;136:779-86.

33 Thompson JS, Hodge DS, Borczyk AA. Rapid biochemical test to identify Verocytotoxin-positive strains of Escherichia coli serotype $0157, f$ Clin Microbiol $1990 ; 28: 2165-8$ 